\title{
Analyzing Driving and Road Events via Smartphone
}

\author{
Nidhi Kalra \\ Computer Science Department \\ PEC University of Technology \\ Sector12, Chandigarh 160012, \\ India
}

\author{
Gunjan Chugh \\ Computer Science \\ Department \\ PEC University of Technology \\ Sector12, Chandigarh 160012, \\ India
}

\author{
Divya Bansal \\ Computer Science \\ Department \\ PEC University of Technology \\ Sector12, Chandigarh 160012, \\ India
}

\begin{abstract}
Travelling in India by road is considered dangerous as traffic conditions are chaotic, the drivers drive recklessly, and the roads are poorly maintained. Therefore, there is need to monitor driver behavior and road condition regularly. In this work, we developed a method that collects data using accelerometer sensor present in Smartphone and analyze driving events and road anomalies. This paper presents the patterns of driving events, such as left turn, right turn, sudden braking and sudden forward acceleration, and road conditions, such as pothole, bump and rough patch, detected in the data set. This work will help in analyzing driving behavior and road anomalies to ensure driver safety and maintenance of roads.
\end{abstract}

\section{General Terms}

Driving Events, Road Anomalies

\section{Keywords}

Left Turn, Right Turn, Sudden Braking, Sudden Forward Acceleration, Pothole, Bump and Rough patch.

\section{INTRODUCTION}

Although there are various transport modes but road transportation mode still plays a major role in people lives. In this busy world all people in hurry to reach their destination as quickly as possible which further leads them to drive fast, recklessly, without obeying the traffic rules which further lead them to accidents sometimes also to their loss of lives. In India potholes, shards of glass, speed breakers, cow dung, garbage, mud pits and many other things that may be found on the road and also less attention is given to the maintenance of roads. So there arises the need to monitor the driving style and road anomalies to ensure driver safety and for the maintenance of roads.

Previously a lot of work is done in this field but researchers mainly focused on monitoring either driver behavior or road conditions using specialized hardware deployed inside the car [1] [2] [3] [4] [5] [6] or roadside which is expensive and also requires maintenance. Our method, contrary from previous work, use accelerometer sensor of Smartphone to observe both the pattern of driver's driving style and road anomalies. Smartphone based approach is considered good as it is inexpensive, portable, requires less maintenance and also relying to the fact that many people already own it.

This paper is organized as follows, section 2 discusses already present techniques for detecting driving events detection and road anomalies, section 3 contains experimental setup for data collection followed by data preprocessing, section 4 discusses the different patterns analyzed of various driving events and road anomalies, and section 5 provides the conclusion.

\section{RELATED WORK}

An android application is developed by Chirgupta et al [7] to give rating to the driver. They used accelerometer sensor, GPS sensor and camera for video recording. They specified range for safe acceleration/deceleration and specified when the data of accelerometer exceed these safe limit, there is an occurrence of event. In [8] Johnson et al proposed a system known as MIROAD: A Mobile- Sensor-Platform for Intelligent Recognition of Aggressive Driving categorized driving style into normal, aggressive and very aggressive. Multiple sensors are used such as (accelerometer, gyroscope, magnetometer, GPS, video) of I phone 4 and fused related data into a single classifier based on Dynamic Time Warping (DTW) algorithm. Their system gives audible feedback if drive drives rashly. A android application is developed by $\mathrm{P}$. Singh et al. [9], which uses data from accelerometer, GPS and microphone. They combined data with sound patterns to detect rash driving behavior and road conditions. Nericell [10] uses accelerometer, microphone, GSM radio and GPS sensors present in smart phones to detect potholes, braking, bumps and honks. To make the system energy efficient high energy consuming sensor (GPS, microphone) is activated by a low energy consuming sensor (accelerometer, cellular radio) by the means of triggered sensing. Mednis et al., [11] proposed a system for detection of potholes in real time which uses Android OS based Smart-phones having accelerometer sensor. Their system is able to detect real time potholes and also data for offline post-processing. Four algorithms have been proposed for detection of potholes. The first two algorithms (Z-THRESH and Z-DIFF) are for real time detection and the other two (STDEV (Z) and G-ZERO) are used for off-line post-processing of data. Wolverine [12] uses accelerometer sensor data for detection of bumps and brakes. They also proposed a algorithm for reorienting the mobile phone device to align it with vehicle axis, as phone can be in any arbitrary location inside the vehicle. K-means clustering is used to classify the sensor data into two classes which is smooth or bumpy (for bump detection) and brake or not (for braking detection). Support Vector Machine (SVM) is trained for classification of data points during test phase for vehicle state prediction. This system gives $10 \%$ false negative rate for bump detection and $21.6 \%$ false negative rate and $2.7 \%$ false positive rate for braking detection.

\section{EXPERIMENTAL SETUP}

\subsection{Data Collection}

Data is collected from accelerometer sensor present in Smartphone using the app "Data_Collection" as shown in Fig 2. All values of accelerometer sensor i.e. $\mathrm{x}, \mathrm{y}$ and $\mathrm{z}$ are fetched from Smartphone's internal services and displayed to the user. Data is collected at time intervals i.e. $200 \mathrm{~ms}(5$ readings per second). While collecting the data, the phone is 
placed at fixed location at dashboard of vehicle in portrait mode as shown in Fig 1.

$\mathrm{X}$-axis points toward right along the smaller side,

$\mathrm{Y}$-axis points up along longer edge and

$\mathrm{Z}$-axis points towards the sky perpendicular to the plane of the front face of the screen.

The all values are stored in phone's memory in .txt format. The output of the data collection segment is raw values of $x, y$ and $\mathrm{z}$ axis of accelerometer sensor.

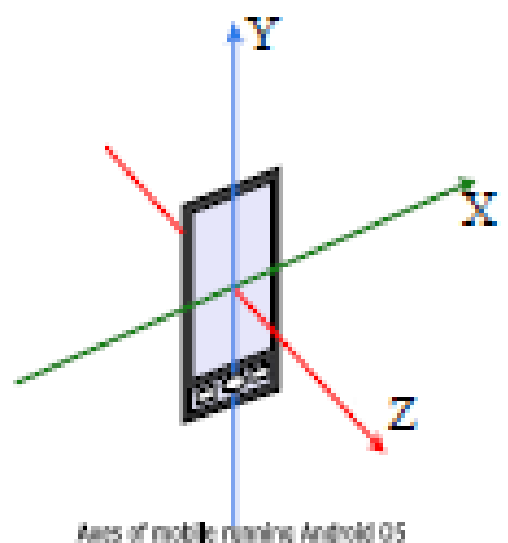

Fig 1: Orientation of Accelerometer in Smartphone

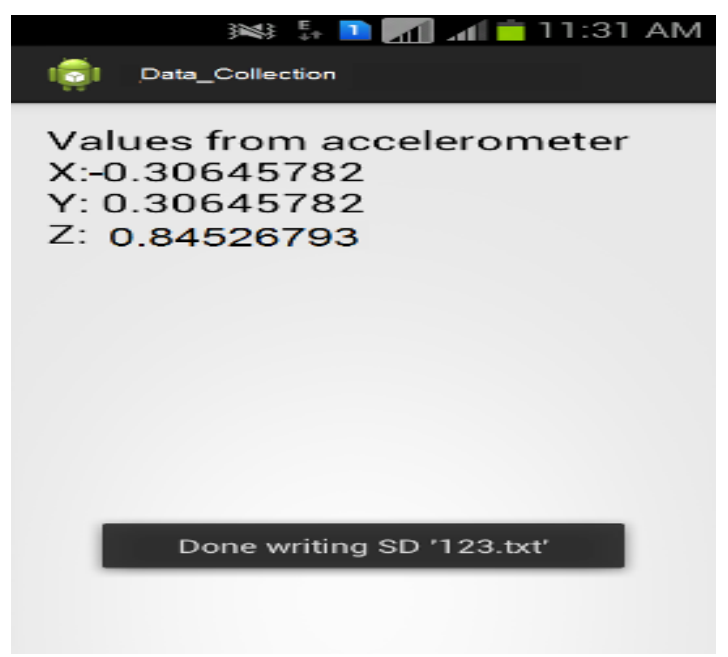

Fig 2: Data Collection App User Interface

\subsection{Data Preprocessing}

The output of data collection module is fed into preprocessing module. There are certain problems in raw data:

- Wide range of values observed

- Data is noisy

- Data shows False Positive and False Negative Rate as shown in Fig 3.

- Data is not in normalized form

- Data is device dependent as different devices have different levels of sensitivity and show different readings for the same event, so it is difficult to set a common threshold for every phone.

Therefore, there was a need to do some preprocessing of data. The preprocessing module provides the gradient data as shown in Table.1. The feature used for classification is difference between the consecutive values.

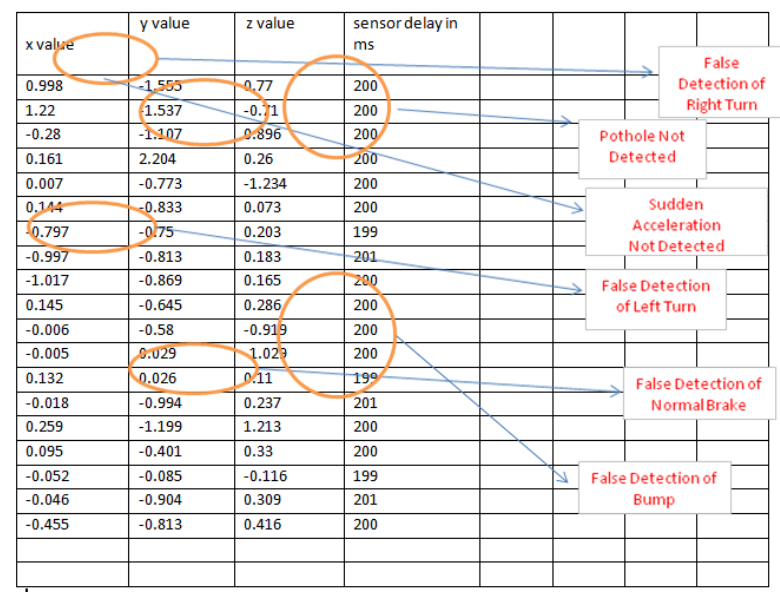

Fig 3: Raw Data showing False Positive and False Negative

Table 1. Gradient Data

\begin{tabular}{|c|c|c|}
\hline X_value & y_value & z_value \\
\hline 1.242 & 0.018000007 & -1.18 \\
\hline-1.5 & 0.42999995 & 1.006 \\
\hline 0.44099998 & 0.24799997 & -0.33600003 \\
\hline-0.154 & 0.086000025 & -0.025999993 \\
\hline 0.137 & -0.060000002 & -0.161 \\
\hline-0.151 & 0.083000004 & 0.13 \\
\hline 0 & -0.06300002 & -0.019999996 \\
\hline-0.137 & -0.055999994 & -0.017999992 \\
\hline 0.28899997 & 0.22400004 & 0.12100001 \\
\hline-0.151 & 0.065 & -0.16700001 \\
\hline 0.001 & 0.60899997 & -0.148 \\
\hline 0.137 & -0.002999999 & 0.139 \\
\hline-0.15 & -0.69100004 & 0.127 \\
\hline 0.277 & 0.066000044 & -0.024000004 \\
\hline-0.164 & 0.19799998 & 0.11700001 \\
\hline-0.147 & 0.31599998 & -0.446 \\
\hline 0.006000001 & -0.819 & 0.42499998 \\
\hline-0.409 & 0.09099996 & 0.10700002 \\
\hline 0.458 & 0.08100003 & -0.593 \\
\hline 0.68899995 & 1.038 & 0.982 \\
\hline-0.34499997 & 1.9000001 & -1.596 \\
\hline-0.311 & -2.9780002 & 0.21700001 \\
\hline
\end{tabular}




\section{PATTERNS OBSERVED}

After analyzing the gradient data and doing various experiments, patterns of various driving events and road anomalies are analyzed and threshold is determined. Table 2 shows the threshold values of various events.

Table 2.Threshold for Driving Events and Road Anomalies Detection

\begin{tabular}{|c|c|c|}
\hline Event & $\begin{array}{l}\text { Axis Used for } \\
\text { Detection }\end{array}$ & Threshold \\
\hline $\begin{array}{l}\text { Normal } \\
\text { Braking }\end{array}$ & $\begin{array}{l}\text { y-axis(in } \\
\text { negative } \\
\text { direction) }\end{array}$ & -1 to -3 \\
\hline $\begin{array}{l}\text { Sudden } \\
\text { Braking }\end{array}$ & $\begin{array}{l}\text {-axis(in } \\
\text { negative } \\
\text { direction) }\end{array}$ & $<-3$ \\
\hline $\begin{array}{l}\text { Sudden } \\
\text { Forward } \\
\text { Acceleration }\end{array}$ & $\begin{array}{l}\text { y-axis(in positive } \\
\text { direction) }\end{array}$ & $>3$ \\
\hline Left Turn & $\begin{array}{l}x \text {-axis(in } \\
\text { negative } \\
\text { direction) }\end{array}$ & $<-1$ \\
\hline Right Turn & $\begin{array}{l}\text { x-axis(in positive } \\
\text { direction) }\end{array}$ & $>1$ \\
\hline Pothole & $\begin{array}{l}\text { z-axis (change in } \\
\text { value from } \\
\text { positive to } \\
\text { negative) }\end{array}$ & \pm 1.5 \\
\hline Bump & $\begin{array}{l}\text { z-axis (change in } \\
\text { value from } \\
\text { negative to } \\
\text { positive) }\end{array}$ & \pm 1.5 \\
\hline Rough Road & $\begin{array}{l}\text { z-axis } \\
\text { (continuous } \\
\text { occurrence of } \\
\text { potholes) }\end{array}$ & \pm 1.5 \\
\hline
\end{tabular}

The following patterns were observed:

\subsection{Straight Driving and Smooth Road}

When the vehicle is at rest or moving straight on a smooth road, then also there will be slight deflection in the value of accelerometer.Therefore, the values of $\mathrm{x}, \mathrm{y}$ and $\mathrm{z}$ axis of accelerometer within the range from ' $-1 \mathrm{~g}$ to $1 \mathrm{~g}$ ' are considered stable as no event is detected within this range as shown in Fig 4.

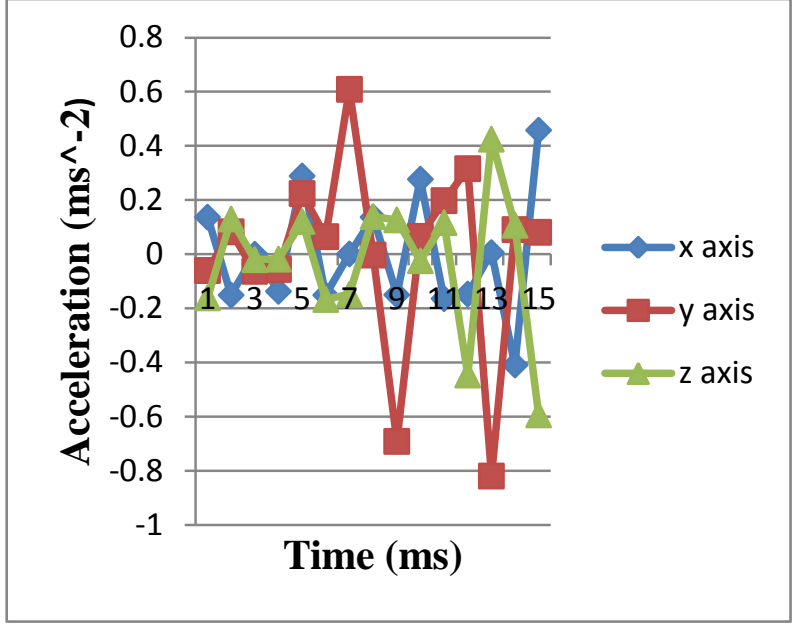

Fig 4: Pattern for Straight Driving and Smooth Road

\subsection{Left Turn}

Left turn will be determined by the change in acceleration in $\mathrm{x}$-axis. If there is sudden decrease in magnitude value of $\mathrm{x}$ axis in negative direction, it indicates left-turn as shown in Fig 5.

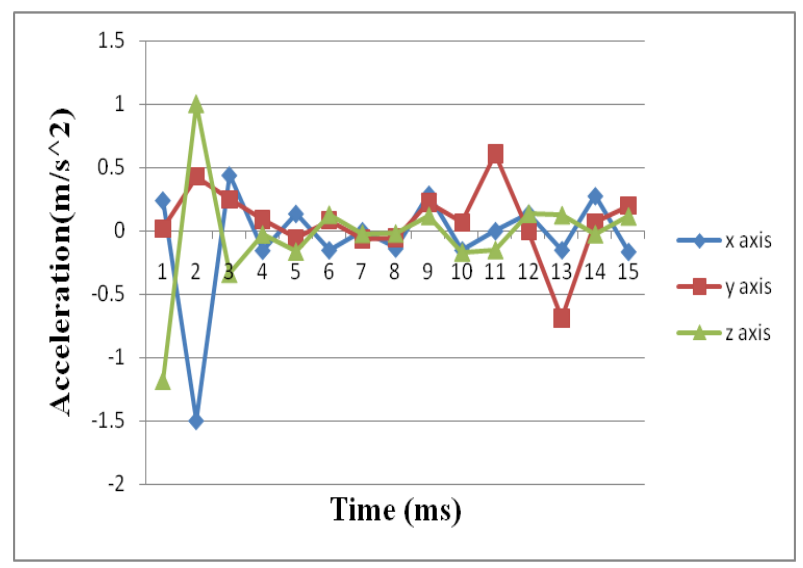

Fig 5: Pattern for Left Turn

\subsection{Right Turn}

Right turn will be determined by the change in acceleration in $\mathrm{x}$-axis. If there is sudden increase in magnitude value of $\mathrm{x}$ axis in positive direction, it indicates right-turn as shown in Fig 6.

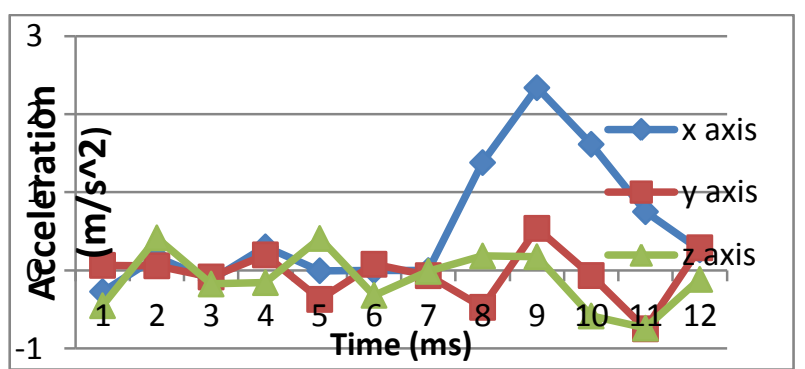

Fig 6: Pattern for Right Turn 


\subsection{Normal Braking}

Braking event will be determined by the change in acceleration in y-axis. In this there is sudden decrease in magnitude value of $y$ - axis in negative direction and also there is slight deflection in $\mathrm{x}$ - axis and $\mathrm{z}$ - axis. Frequent braking can be due to various reasons for e.g. heavy congestion, bumpy road or environmental factors such as rainfall as shown in Fig 7.

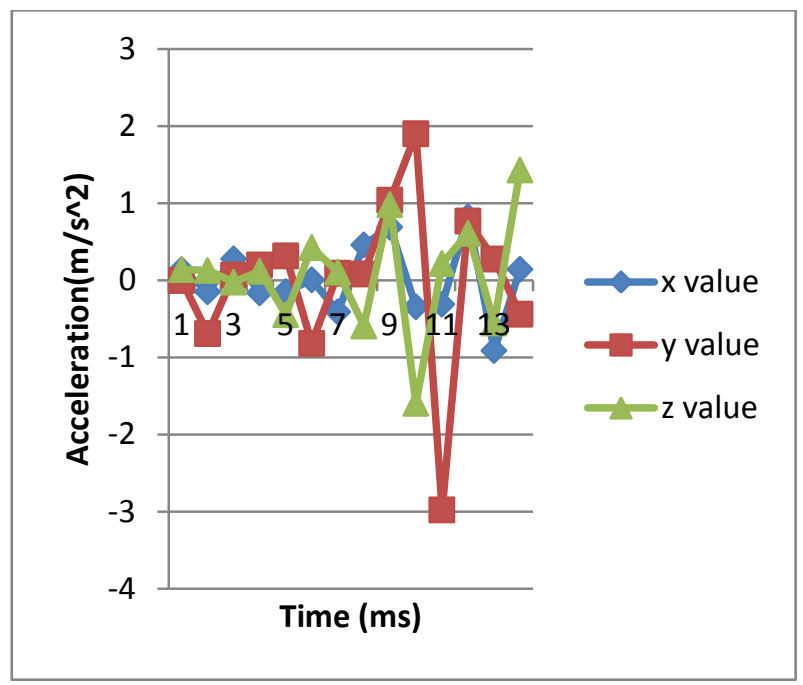

Fig 7: Pattern for Normal Brake

\subsection{Sudden Braking}

Sudden Braking event will be determined by the change in acceleration in y-axis. In this there is very large decrease in the $y$-axis in negative direction or we can say when there is deflection in y axis in negative direction <-3, sudden braking event occurred as shown in Fig 8.

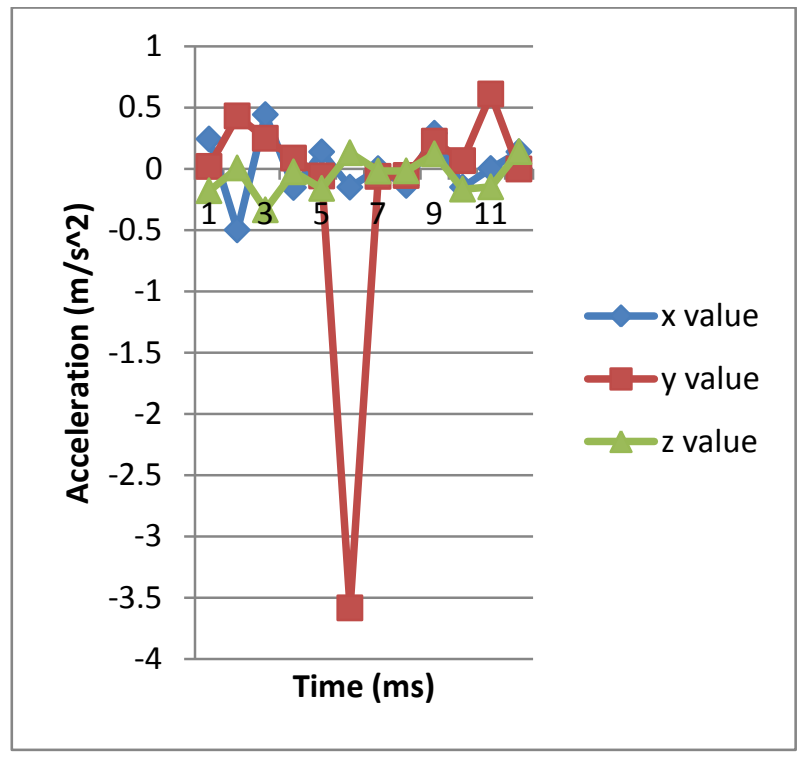

Fig 8: Pattern for Sudden Brake

\subsection{Sudden Forward Acceleration}

Sudden Forward Acceleration will be determined by the change in acceleration in $\mathrm{y}$-axis. In this there is sudden increase in magnitude value of $y$ - axis in positive direction and there is some deflection in $\mathrm{x}$ - axis and $\mathrm{z}$ - axis as shown in Fig 9.

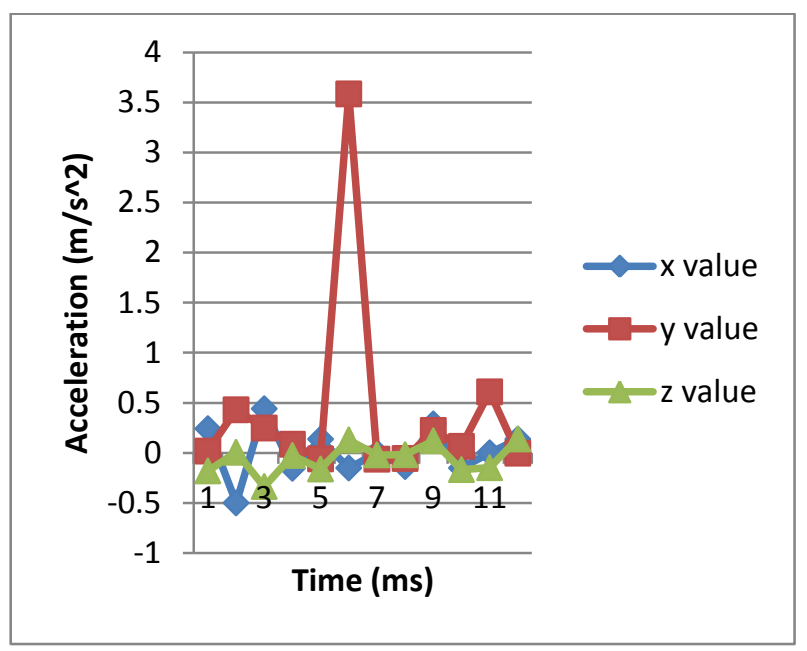

Fig 9: Pattern for Sudden Forward Acceleration

\subsection{Pothole}

The pothole will be indicated with the change in the value of $\mathrm{z}$-axis. If there is no (or slight) deflection in the value of $\mathrm{z}$ axis, then it is smooth road (no bump or pothole) and if there is any deflection, a bump or pothole is detected. If the magnitude of value of z-axis deflects firstly in positive direction and then towards negative direction, with slight deflection in $\mathrm{x}$ and $\mathrm{y}$ value, then it will indicate a pothole as shown in Fig 10.

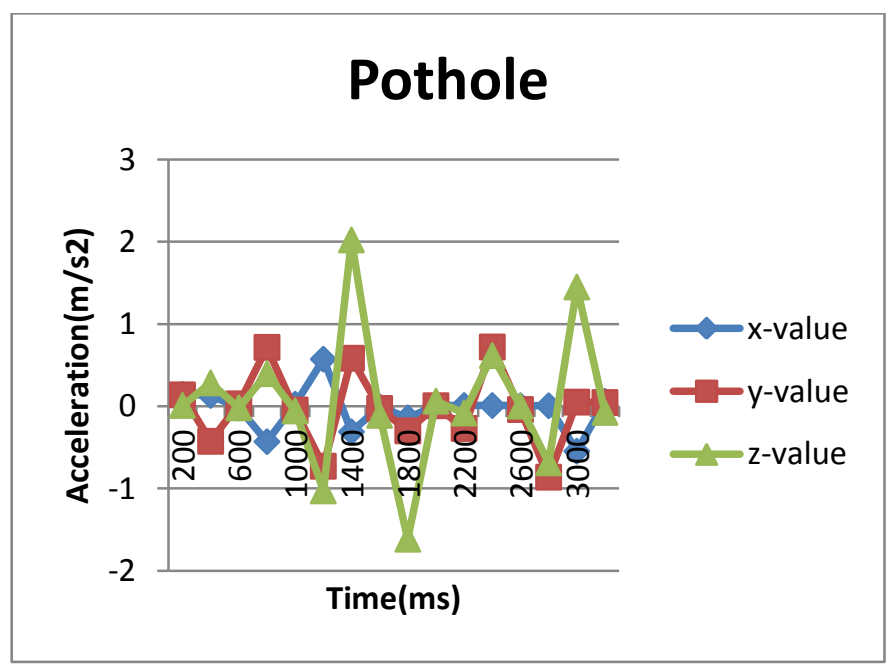

Fig 10: Pattern for Pothole

\subsection{Bump}

The bump (speed breaker) will be indicated with the change in the value of z-axis. If there is no (or slight) deflection in the value of $\mathrm{z}$-axis, then it is smooth road (no bump or pothole) and if there is any deflection, a bump or pothole is detected. If the magnitude of value of z-axis deflects firstly in negative direction and toward positive direction, with slight deflection in $\mathrm{x}$ and $\mathrm{y}$ value, then it will indicate a bump as shown in Fig 11 . 


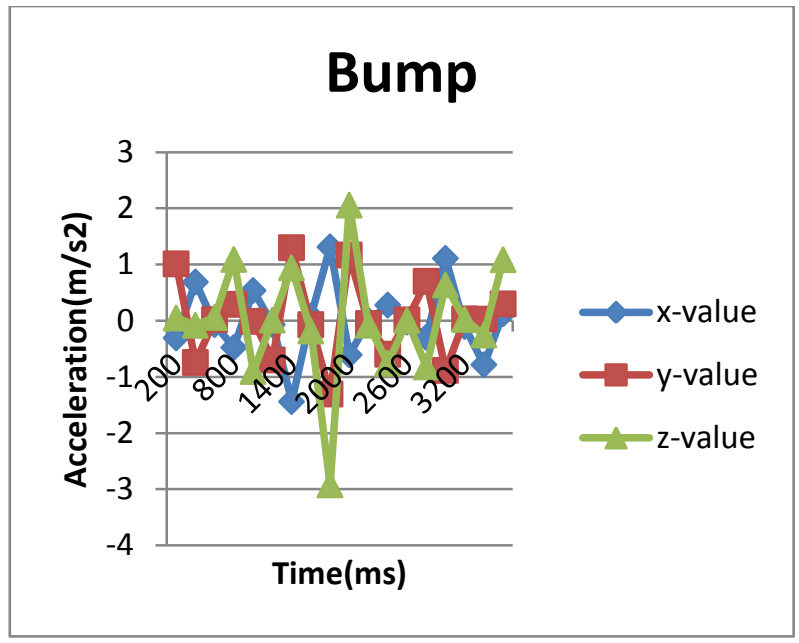

Fig 11: Pattern for Bump

\subsection{Rough Road}

Rough road is indicated by a continuous occurrence of potholes. If there is continuous zigzag motion in the values of $\mathrm{z}$-axis, then it is considered as rough patch of road as shown in Fig 12.

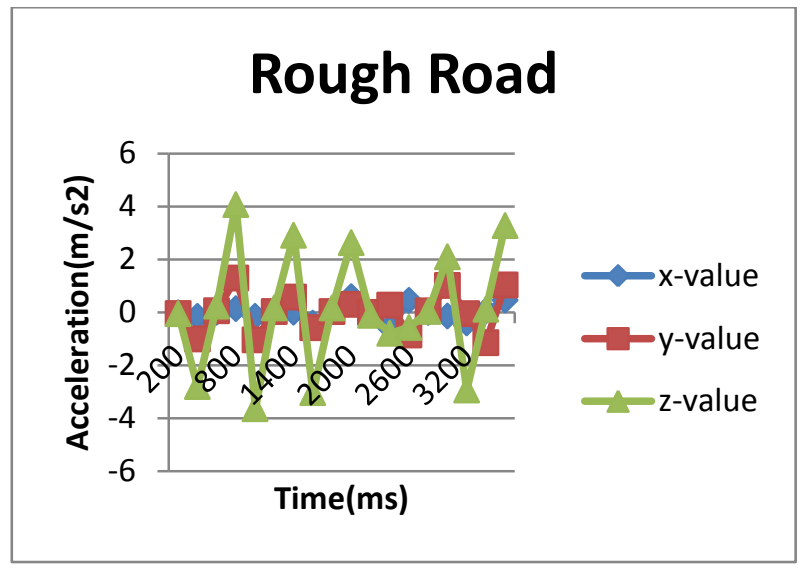

Fig 12: Pattern for Rough road

\section{CONCLUSION}

This work shows pattern of driving events and road anomalies using Smartphone. Data from accelerometer sensor present in Smartphone is fetched and by doing experimental analysis various threshold are set and used for distinguishing and classifying various driving events and road anomalies. In the future work there is a scope of feeding these patterns into machine learning classifier to find out the patterns from collected data and to classify them. Also these patterns can be helpful in monitoring driver behavior and road conditions thereby lower the risk of road accidents. These patterns can be integrated to develop an android application which can help person in real time when he or she drives rashly or in case of bad road.

\section{REFERENCES}

[1] Hull, B., Bychkovsky, V., Zhang, Y., Chen, K., Goraczko, M., Miu, A., \& Madden, S., 2006, CarTel: a distributed mobile sensor computing system, Proceedings of the 4th international conference on Embedded networked sensor systems, Boulder, Colorado, USA, October ,2006,pp. 125-138. ACM.

[2] De Silva, G. D., Perera, R. S., Laxman, N. M., Thilakarathna, K. M., Keppitiyagama, C. I., \& De Zoysa, K., Automated Pothole Detection System, International Conference on Advances in ICT for Emerging Regions, Colombo, Sri Lanka, 2013.

[3] Eriksson, J., Girod, L., Hull, B., Newton, R., Madden, S., \& Balakrishnan, H., The pothole patrol: using a mobile sensor network for road surface monitoring, Proceedings of the 6th international conference on Mobile systems, applications, and services, Breckenridge, Colorado, USA, June 17-20, 2008,pp. 29-39. ACM.

[4] Chen, K., Lu, M., Fan, X., Wei, M., \& Wu, J., 2011, Road condition monitoring using on-board Three-axis Accelerometer and GPS Sensor, In Communications and Networking in China (CHINACOM), 2011 6th International ICST Conference on August,2011,pp. 10321037. IEEE

[5] Zhang, Y., Lin, W. C., \& Chin, Y. K. 2010, A patternrecognition approach for driving skill characterization, Intelligent Transportation Systems, IEEE Transactions on, April 11,2010,pp. 905-916.

[6] Sathyanarayana, A., Boyraz, P., \& Hansen, J. H. , 2008 Driver behavior analysis and route recognition by hidden Markov models. In Vehicular Electronics and Safety, 2008. ICVES 2008. IEEE International Conference on September 2008, pp. 276-281. IEEE.

[7] Chigurupa, S., Polavarap, S., Kancherla, Y., Nikhath, K.A.: Integrated Computing System for measuring Driver Safety Index. In: International Journal of Emerging Technology and Advanced Engineering, ISSN 2250-2459, Volume 2 (2012)

[8] Johnson, D.A., Trivedi, M.M.: Driving Style Recognition using a smartphone as a sensor platform. In: IEEE 14th International Conference on Intelligent Transportation system, October(2011)

[9] Singh, P., Juneja, N., Kapoor, S.: Using mobile phone sensors to detect driving behavior. In: Proceedings of the 3rd ACM Symposium on Computing for Development, ACM (2013)

[10] Mohan, P., Padmanabhan, V. N. and Ramjee R.; Nericell: rich monitoring of road and traffic conditions using mobile smartphones. In: 6th ACM conference on Embedded network sensor systems, SenSys '08, pp 323 336. ACM, New York, NY, USA (2008).

[11] Mednis, A., Strazdin, G., Zviedris, R., Kanonirs, G., and Selavo, L.; Real time pothole detection using android smartphone with accelerometers. In: International Conference on Distributed Computing in Sensor Systems and Workshops (DCOSS). IEEE (June 2011).

[12] Bhoraskar, R., Vankadhara, N., Raman, B., Kulkarni P.; Wolverine: Traffic and Road Condition estimation using Smartphone Sensors. In: Fourth International Conference on Communication Systems and Networks (COMSNETS). IEEE (January 2012). 